\title{
Bacteriological Spectrum of Sputum in Acute Exacerbation of Chronic Obstructive Pulmonary Disease (COPD)
}

\author{
Tahera Khatun ${ }^{*}$, Aparna Das ${ }^{2}$, Gobinda Chandra Banik ${ }^{3}$, Uma Dhar $^{4}$, Mohiuddin Sharif5 $^{5}$ Kayanat Yeasmin ${ }^{6}$, \\ Suchismita Debnath7
}

\begin{abstract}
Background: Acute Exacerbation of COPD (AECOPD) is natural course of Chronic Obstructive Pulmonary Disease(COPD), where bacterial infection is the major cause. Bacterial infection worse the sign symptoms of the patients and ultimately increase morbidity \& mortality.

Methods: This is a cross-sectional observational study. 100 clinically diagnosed cases of AECOPD aged between 18 and 85 years were included in the study. The sputum specimen was processed by conventional methods and subjected to culture and sensitivity in standard laboratory.

Results: The prevalence of AECOPD was more common in age group of fifty-six to sixty- five years (38\%) with ratio between male and female of 4.6:I.The prevalence of Gram-negative bacteria was $65.21 \%$ and Gram-positive bacteria was $34.79 \%$. K. pneumoniae was the commonest bacteria isolated (36.95\%) followed by P. aeruginosa (2I.73\%), S. aureus (I5.2I), S. pneumoniae (I0.86), S. pyogenes and E. coli (6.52\%), MRSA (2.17\%). The drug sensitivity reveals that $79.55 \%$ of the isolates were sensitive to Amikacin (79.55\%) followed by Azithromycin (70.45\%), Amoxy clavulanic acid (68.18\%) and Ciprofloxacin (63.63\%).

Conclusion: Repeated exacerbation and hospital admission leads to a major impact on the quality of life of patients with COPD.Antibiogram helps in screening resistant pathogens and prescribing right treatment protocol.
\end{abstract}

Key words: AECOPD, Bacteria, antibiogram.

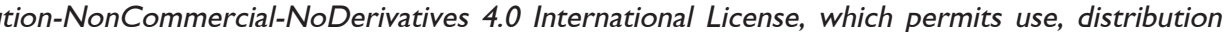
and reproduction in any medium, provided the original work is properly cited, is not changed in any way and it is not used for commercial purposes.

Received: 08, June, 202I;

Accepted: 09, November, 2021

\section{Introduction}

Chronic Obstructive Pulmonary Disease (COPD) is a major health problem all over the world. It is highly prevalent, undertreated and under perceived disease. The number of individuals affected has grown since $1980 \&$ this increase is expected to continue during next decades. ${ }^{1}$ COPD is characterized by intermittent acute exacerbation associated with worsening of symptoms and lung function. Acute

1. Medical Officer, Dhaka Medical College Hospital, Dhaka

2. Associate Professor of Medicine, Dhaka Medical College Hospital, Dhaka

3. Associate Professor of Medicine, Dhaka Medical College Hospital, Dhaka

4. MD Respiratory Medicine (phase B) student, BSMMU, Dhaka.

5. Medical Officer, Dhaka Medical College Hospital, Dhaka

6. Assistant surgeon, Upazila Health Complex, Brahmanbaria.

7. Medical Officer, BSMMU, Dhaka.

*Corresponding author: Dr.Tahera Khatun, Medical Officer, Department of Medicine, Dhaka Medical College Hospital, Email: tahera.ssmc@gmail.com, Cell: +8801718735565 exacerbations reduce quality of life, speed disease progression. Bacterial infections in patients of COPD is still continued as a health problem especially in developing countries with high morbidity and mortality often due to respiratory failure. COPD related death is probably underestimated because of the difficulties associated with identifying the precise cause of death. ${ }^{2}$ Comorbidities such as cardiovascular diseases and lung cancer are also major causes and, in mild to moderate COPD, are the leading causes of mortality. Smoking cessation improves survival in COPD patients, as well as reduce risk of death from other comorbid conditions. $^{3}$

COPD is the most common respiratory disorder encountered in clinical practice and accounts for $1-2.5 \%$ admissions in hospitals all over Bangladesh ${ }^{4}$. Acute exacerbation showed a hospital mortality rate of $24 \%$ if the patient required ICU admission. This mortality rate increased to $30 \%$ if the patient was above 65 years. ${ }^{5}$ It is both a rural and urban health problem, the prevalence varying from $1 \%$ in urban nonsmoker to $21 \%$ in rural smokers. This increasing 
prevalence and mortality have led to an extensive research related to these diseases. Much has been learnt about pulmonary response to irritants, particulate antigenic materials and infectious agents. ${ }^{4}$ COPD is fourth most common cause of death in countries like USA. Both the prevalence and mortality from this disease has been increasing worldwide.

Several potential contributions of bacterial infection to the etiology, pathogenesis and clinical course of COPD can be identified. Three classes of pathogens have been implicated as causing AECOPD by infecting the lower respiratory tract: respiratory viruses, atypical bacteria, and aerobic Gram-positive and Gram-negative bacteria. The relative contributions of these three different classes of pathogens may change depending on the severity of the underlying obstructive airway disease. Such changes may also happen within a class, especially for bacterial pathogens. $^{7}$

In last decade with the increasing use of fiber optic bronchoscopy, newer sampling methods like trachea bronchial aspirated sample (TBAS), bronchoalveolar lavage fluid (BALF), and protected specimen brushing (PSB) have emerged. ${ }^{8}$ This has renewed interest in the area of bacteria and COPD, and this should lead to a precise delineation of the contribution of bacterial infection to the disease. ${ }^{9}$ In COPD, acute exacerbation is the common problem during natural course. The study is carried out to find out the bacterial etiology in the course of disease and their drug sensitivity pattern.

\section{Materials and Methods}

All hospitalized patients diagnosed with AECOPD admitted in the Department of medicine, Dhaka Medical College Hospital, Dhaka from July 2017 to December 2017, were evaluated. The study was carried out COPD was diagnosed according to the Global Initiative for Obstructive Lung Disease (GOLD) guidelines. AECOPD was assumed when a patient presented with at least two of the three following symptoms: (a) worsening dyspnea, (b) increased cough, and (c) increased sputum production. Written informed consent was obtained from all the study participants. This study was approved by the institutional ethics committee.

Patients were excluded for the study if (1) they had an outpatient status; (2) received antibiotic within last 48 hours of hospital admission; (3) Evidence of bronchiectasis, pneumonia or asthma; (4) other known chronic respiratory disorder; (5) active malignancy; (6) immunosuppression; (7) Absence of adequate sputum sample; and 8) Patient on mechanical ventilation. Patients were included only once in study even if they hospitalized frequently during study period.

Variables included in the study were age, gender, smoking history or exposure to indoor smoke, signs at presentation and nature of sputum. After giving an informed consent, all patients were subjected to detailed history and both general and systemic examination. After clinical examination all patients underwent a chest radiography, complete blood counts, differential blood count, oxygen saturation by pulse oximetry.

Early morning deep coughed sputum sample was collected from all participants according to standard guideline. Within 24 hours of admission, patients were asked to collect sputum into a universal sterile wide mouthed container with a screw cap. Sputum samples were examined for physical appearance, gram stain, acid fast bacilli smear, culture for bacterial organism and drug sensitivity testing. All data was entered and analyzed by SPSS software program. Categorical variables will be reported as percentage.

\section{Results}

The individual bacterial isolates and their sensitive pattern to various antibiotics were also recorded in all one hundred (100) patients.

Table 1 : Age and Sex distribution

\begin{tabular}{lccc}
\hline Age & Male & Female & Percentage \\
\hline $18-35$ & 0 & 0 & $0 \%$ \\
$36-45$ & 8 & 0 & $8 \%$ \\
$46-55$ & 15 & 3 & $18 \%$ \\
$56-65$ & 30 & 8 & $38 \%$ \\
$66-75$ & 19 & 4 & $23 \%$ \\
$76-85$ & 10 & 3 & $13 \%$ \\
\hline Total & 82 & 18 & $100 \%$ \\
\hline
\end{tabular}

Out of one hundred (100) patients, clinically diagnosed as AECOPD, eighty-two (82\%) were males and eighteen (18\%) were females. The ratio between male and female is $4.6: 1$. Here, fifty-six to sixty-five years $(38 \%)$ is most common age group. 


\section{Bacteriological profile}

Out of a total 100 cases, forty-four (44\%) were positive for pathogenic bacteria and fifty-six (56\%) were non-pathogenic. (Figure 1)

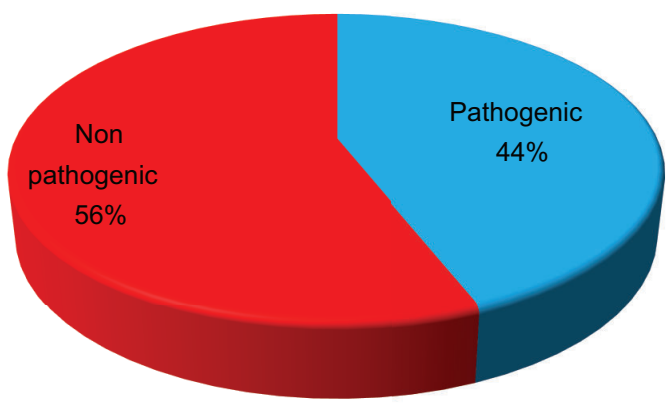

Figure 1: Involvement of pathogens in $A E C O P D$

Out of forty-four (44) cases pathogenic bacteria isolated, forty-two (42) had single microbial infections and two (2) had poly-microbial infections. (Figure 2)



Figure 2: Distribution of isolates in AECOPD

Among total forty-six $[42+(2 \times 2)]=46$ pathogenic microbial, thirty $(65.21 \%)$ were Gram-negative bacteria and sixteen $(34.79 \%)$ were Gram-positive bacteria. (Figure 3)



Figure 3: Type of bacteria isolated in AECOPD
Out of forty-two (42) single pathogenic bacteria, K. pneumoniae was the commonest (36.95\%) followed by $P$. aeruginosa (21.73\%), S. aureus(15.21\%), S. pneumoniae $(10.86 \%)$, S. pyogenes $(6.52 \%)$. (Table 2$)$

Out of forty-four (44) culture report of sputum, two (2) samples showed more than one isolates. The pattern of poly-microbial infection is as follows:

(K. pneumoniae + S. aureus)

(K. pneumoniae + S. pneumoniae).

Table-2 : Bacterial Strains Isolated from Sputum Samples

\begin{tabular}{llcc}
\hline Sl No. & Name of the organism & Number & Percentage \\
\hline 1. & K. pneumoniae & 17 & $36.95 \%$ \\
2. & P. aeruginosa & 10 & $21.73 \%$ \\
3. & S. aureus & 7 & $15.21 \%$ \\
4. & S. pneumoniae & 5 & $10.86 \%$ \\
5. & S. pyogenes & 3 & $6.52 \%$ \\
6. & E. coli & 3 & $6.52 \%$ \\
7. & MRSA & 1 & $2.17 \%$ \\
\hline & Total & 46 & \\
\hline
\end{tabular}

\section{Antibiotic sensitivity patterns of the isolates}

K. pneumoniae was the most common isolate and was sensitive to Amikacin,, Gentamycin, Ciprofloxacin, Ceftriaxone and Amoxy Clavulanic acid. P. aeruginosa was the next common isolate and was sensitive to Amikacin, Ceftriaxone, Gentamycin and Co-trimoxazole. S. aureus was sensitive to Levofloxacin, Amoxy clavulanic acid, Azithromycin and S. pneumoniae was sensitive to Amoxy Clavulanic acid, Azithromycin, Ceftriaxone and cefuroxime. S. pyogenes was sensitive to Ciprofloxacin, Ceftriaxone, Levofloxacin and Amoxy Clavulanic acid. E. coli was sensitive to Amikacin, Ciprofloxacin, Ceftriaxone and Gentamycin.

Among antibiotics, Amikacin was found highest sensitive followed by Azithromycin (70.45\%), Amoxy Clavulanic acid (68.18\%), Ciprofloxacin (63.63\%) and Gentamycin (56.81\%). (Table 3) 
Table-3 : Antimicrobial Susceptibility pattern of isolated bacteria

\begin{tabular}{llcc}
\hline Sl No. & Antibiotics & No. of Isolates & Percentage \\
\hline 1. & Amikacin & 35 & $79.55 \%$ \\
2. & Amoxy Clavulanic acid & 30 & $68.18 \%$ \\
3. & Cefuroxime & 18 & $40.91 \%$ \\
4. & Ceftriaxone & 19 & $43.18 \%$ \\
5. & Ciprofloxacin & 28 & $63.63 \%$ \\
6. & Co-trimoxazole & 16 & $36.36 \%$ \\
7. & Gentamicin & 25 & $56.81 \%$ \\
8. & Azithromycin & 31 & $70.45 \%$ \\
9. & Levofloxacin & 13 & $29.54 \%$ \\
\hline
\end{tabular}

However, Levofloxacin and Co-trimoxazole were found to be highly resistant $70.45 \%$ and $63.63 \%$ respectively among the drugs used in culture and sensitivity of 46 isolated pathogenic bacteria. (Table 4)

Table - 4 : Resistant pattern among Bacterial isolates

\begin{tabular}{llcc}
\hline S1. No. & Antibiotics & No. of Isolates & Percentage \\
\hline 1. & Amikacin & 9 & $20.45 \%$ \\
2. & Amoxy Clavulanic acid & 14 & $31.82 \%$ \\
3. & Cefuroxime & 26 & $59.09 \%$ \\
4. & Ceftriaxone & 25 & $56.81 \%$ \\
5. & Ciprofloxacin & 16 & $36.36 \%$ \\
6. & Co-trimoxazole & 28 & $63.63 \%$ \\
7. & Gentamicin & 19 & $43.18 \%$ \\
8. & Azithromycin & 13 & $29.54 \%$ \\
9. & Levofloxacin & 31 & $70.45 \%$ \\
\hline
\end{tabular}

\section{Discussion}

Sputum culture is a good and simple tool to study the aetiology \& complications due to bacteria in AECOPD. If done well, it can replace the costlier diagnostic methods like Immunodiffusion. Antibiogram helps in the correct treatment protocol during management of acute exacerbation of COPD. ${ }^{10}$ It also helps in screening resistant pathogens and better drug for treatment, thereby helping to decrease the mortality and morbidity.

This study presented here involved 100 patients with history of acute exacerbation of COPD. They presented following history of increase in sputum volume, increase in sputum purulence \& increase in shortness of breath.
It was observed that AECOPD was prevalent in 35-85 years age group. However, among them, 56-65 years age constituted $38 \%$. This is because it was more commonly seen in patients with advanced lung disease as an expression of deterioration in host defence at the bronchial mucosal level. ${ }^{11}$

COPD was higher in males $82(82 \%)$ than females $18(18 \%)$. This finding is in conformity with the observation of otherworkers. ${ }^{12}$ The male preponderance was seen in our study because most of them were smokers. In non-smokers, especially among women, exposure to indoor air pollution was an important factor. ${ }^{6}$

It is well known that the frequency of infection resulting in acute exacerbation of COPD by various microorganisms varies from one geographical area to another. Out of 100 sputum sample, pathogenic bacteria were found in $44 \%$ of patients with AECOPD. This could be due to declining lung function. $^{13}$

The prevalence of Gram negative isolates was $65.21 \%$, as compared to $34.79 \%$ of gram positive, which was similar to the study conducted previously in China. The Gram negative organisms were more common in the patients with the most severe lung dysfunction, where the Gram positive bacteria predominated in the exacerbations of the patients with the mildest degree of lung function abnormalities. ${ }^{14}$

Among the gram negative isolates, K. pneumonia was the predominant organism isolated $36.95 \%$ followed by $\mathrm{P}$. aruginosa $21.73 \%$, E. coli $6.52 \%$. Gram positive isolates were S. aureus $15.21 \%$, S. pneumoniae $10.86 \%$, S. pyogenes $6.52 \%$, MRSA $2.17 \%$.

Kuwal A conducted a study on Indian patients involving different hospitals all over india and at the end point, pathogenic bacteria were isolated in $47.22 \%$ cases, where Pseudomonas aeruginosa was the commonest bacteria (38.23\%) followed by Klebsiella pneumonia (29.41\%), Staphylococcus areus (23.93\%). ${ }^{15}$

A respiratory study considered bacterial infection is the main cause of exacerbation of COPD where pathogenic bacteria were isolated in $66.4 \%$ episodes of acute exacerbation of COPD. The predominant bacteria were Klebsiella pneumoniae (19.6\%), Pseudomonas aeruginosa (16.8\%) and Haemophilus influenzae (7.5\%), followed by Acinetobacter baumannii (6.9\%), Enterobacter species (6.1\%) and Staphylococcus aureus (6.1\%). The incidence of Streptococcus pneumoniae was $2.4 \% .{ }^{16}$

Another Mexican study where pathogenic bacteria were isolated in $34.28 \%$ cases, it were included Klebsiella pneumoniae(16.94\%), Pseudomonas aeruginosa(16.94\%), 
Acinetobacter baumannii(11.49\%), Streptococcus pneumoniae( $(8.87 \%)$, Staphylococcus areus(7.26\%). ${ }^{17}$ Result of these study is similar to our study as same type of bacteria was isolated.

Majority of isolated bacteria were Gram-negative bacilli viz. Pseudomonas and Klebsiella species. The prevalence of lower airway bacterial colonization in outpatients with stable COPD is high and is mainly due to Gram-negative bacilli like Pseudomonas spp. The greater rate of isolation of pathogenic bacteria in exacerbated COPD than in stable COPD in different studies, supports the pathogenic role of bacteria in a proportion of AECOPD. ${ }^{18}$ Acinobacter, Moraxella catarrhalis, H. influenza, and Enterobacter also contributed in exacerbation of COPD. The frequency of isolating a pathogen was increased with severity of dyspnoea. ${ }^{4}$ In our study, few organisms such as H.influenza, Moraxella catarrhalis, Acinobacter were not isolated as small study sample may be the pitfall.

A journal of microbiology showed that serological evidence of M. pneumoniae infection and/or detection of $\mathrm{M}$. pneumoniae specific antigen were seen in $16 \%$ of the cases. One case with definite evidence of M. pneumoniae infection also had coinfection with Pseudomonas spp. ${ }^{19}$ However, no direct evidence of M. pneumoniae infection was found in their study population as defined by culture isolation or PCR. In conclusion, we can't comment on M. pneumoniae as serological investigations were not included in our study.

Based on the sensitivity pattern, the highest sensitive antibiotic was Amikacin (79.55\%) followed by Azithromycin (70.45\%), Amoxy Clavulanic acid (68.18\%), Ciprofloxacin (63.63\%), Gentamycin (56.81\%), Ceftrioxone (43.18\%). The resistant antibiotics were Levofloxacin $(70.45 \%)$, Cotrimoxazole (63.63\%), Cefuroxime (59.09\%), Ceftriaxone (56.81\%) and Gentamycin (43.18\%). Amikacin was found to be highly sensitive as because the majority of isolated bacteria was gram negative.

Antibiotic sensitivity testing of Turk Thorac J 2018 showed that ciprofloxacin and piperacillin/tazobactum were effective in $79.41 \%$ of isolates followed by gentamycin in $76 \%$. Another study J Int Med Res 2015 documented that K.pneumoniae, P. aeruginosa, A. baumannii and E. coli were susceptible to amikacin and cefoperazone. S. aureus was sensitive to tigecycline, teicoplanin, vancomycin and linezolid but resistant to penicillin and levofloxacin. In our study, the percentage of pathogenic bacteria resistant to antibiotics were more pronounced than other study because of injudicious use of antibiotics in our country.

\section{Conclusion}

AECOPD represents a major health burden which is both economic and social because of the propensity of readmissions that resulting transient or permanent deterioration in quality of life. Good laboratory facilities for proper culture and sensitivity of sputum, guide physicians to choose appropriate antibiotic minimizing AECOPD as well as sharp eye on changing pattern of the isolates.

\section{References}

1. Anthony Seaton, Douglas Seaton, A. Gordon Leitch "Chronic Bronchitis and Emphysema." Chronic obstructive pulmonary diseases 'Crofton and Douglas's Respiratory Disease $5^{\text {th }}$ Ed. Wiley-Blackwell.2008.

2. Catia Saraiva, Djamila Neves, Tiago Abreu, Fatima Rodrigues" Mortality in COPD: role of comorbities" European Respiratory Journal ; 201423 December; 44 (58): 603.

3. Cristine E.Berry \& Robert A. Wise, "Mortality in COPD: Causes, Risk Factors, and Prevention" Original research, 2010 Sep 20; 7(5): 375-382.

4. Non Communicable Disease situation, Strategic plan for surveillance \& prevention of Non-communicable Disease in Bangladesh 2007-2010, Director General of Health Services, Ministry of Health \& Family Welfare; Karuka publication; 2010: 4-5.

5. Michael G. Seneff, Douglas P. Wagner, Randall P. Wagner ; Hospital and 1 year survival of patients admitted to ICU with AECOPD; JAMA; 1995 Dec 20; 274(23):1852-1857.

6. Surinder K, Jindal; Emergence of COPD as an epidemic in India; Ind J Med Res; 2006 Dec 25; 124: 619-630.

7. Sethi, Sanjay "Infectious Etiology of Acute Exacerbations of Chronic Bronchitis.” CHEST; 2000 May; 117(5): 380-385.

8. Soler Nestor, Antoni Torres, Santiago Ewig, Julia Gonzalez, Rosa Celis, Mustafa El-Ebiary ; "Bronchial Microbial Patterns in severe Exacerbations of Chronic Obstructive Pulmonary Disease (COPD) Requiring Mechanical Ventilation." A.M.J.Resp. Crit Care Med; 1997 Nov 11; 157(5): 1498- 1505.

9. Sethi Sanjay, Timothy F. Murphy. 2001 "Bacterial Infection in Chronic Obstructive Pulmonary Disease in 2000 : a State - of - the - Art Review"; Clinical Microbiology Reviews; 2000 Dec 18; 14(2): 336-363.

10. S R Kamat, A P Mehta, V B Doshi, S Walimbe, V V Jayakar V; "a Comparative Study of various Antibacterial Drugs in Lower Respiratory Tract Infections and Pneumonias in Adults"; JAPI; 1983 Apr ; 31(4):209-215.

11. Eller Jorg, Anja Ede, Tom Schaberg, Michael S.Niederman; "Infective Exacerbaions of Chronic Bronchitis: Relation 
between Bacteriologic Etiology and Lung Function" CHEST; 1998 June; 113(6):1542-1548.

12. Jindal S.K., A.N. Aggarwal, D. Gupta. "A Review of Population Studies from India to Estimate National Burden of Chronic Obstructive Pulmonary Disease and Its Association with Smoking." Indian J Chest Dis. Allied Sci. ; $2001 ; 43: 139-147$.

13. N Roche, B Kouassi, A Rabbat, A Mounedji, C Lorut, G Huchon; Yield of sputum microbiological examination in patients hospitalized for exacerbations of COPD with purulent sputum; respiration; 2006 Dec ; 74(1):19-25.

14. Niederman Michael S. "antibiotic Therapy of Exacerbations of Chronic Bronchitis." Seminars in Resp. Inf; 2000 Mar 01; 15(1):59-70.

15. Kuwal A, Joshi V, Dutt N, Singh S, Purohit G; A Prospective Study of Bacteriological Etiology in Hospitalized Acute Exacerbation of COPD Patients: Relationship with Lung Function and Respiratory Failure; Turk Thorac J; 2018 Jan; 19(1):19-27
16. Sheng-Hsiang Lin, Ping-Hung Kuo, Po-Ren Hsueh, Pan-Chyr Yang; Sputum bacteriology in hospitalized patients with acute exacerbation of chronic obstructive pulmonary disease in Taiwan with an emphasis on Klebsiella pneumoniae and Pseudomonas aeruginosa; Respirology; 2007 Jan;12(1): 81-7.

17. Xiuqing Ma, Junchang Cui, Jing Wang, Yan Chang, Qiuhong Fang, Changqing Bai, Xiumei Zhou; Multicentre investigation of pathogenic bacteria and antibiotic resistance genes in Chinese patients with acute exacerbation of chronic obstructive pulmonary disease; J Int Med Res. ; 2015 Oct; 43(5):699-710

18. M R Bari, M M Hiron, S M Zaman, M M Rahman, K C Ganguly; Microbes responsible for acute exacerbation of COPD; Mymensingh Med J. ; 2010 Oct; 19(4):576-85.

19. Mandira V Basil, Shailendra K D Dwivedi, Krishna Kumar, Rakesh Pathak, Ritika Rastogi, S S Thukral; Role of Mycoplasma pneumoniae infection in acute exacerbations of chronic obstructive pulmonary disease; J Med Microbiol ;2009 Mar; 58 (3):322-326. 